\title{
EDITORIAL
}

\section{In Through the Out Door: Tips and Tricks for Giving Retrograde Cardioplegia}

\author{
Stephen Derryberry, MD, ${ }^{1}$ Curt Tribble, $M^{2}$
}

${ }^{1}$ General Surgery Resident at Vanderbilt University Medical Center, Nashville, Tennessee, USA; ${ }^{2}$ Division of Cardiac Surgery, University of Virginia Health System, Charlottesville, Virginia, USA

\section{INTRODUCTION}

Antegrade cardioplegia delivery is the most commonly used delivery route. It is given into the aortic root or directly into the coronary ostia. However, there are many reasons to consider using the retrograde delivery of cardioplegia. This treatise will review the background and techniques for delivering retrograde cardioplegia.

\section{WHY IS RETROGRADE PERFUSION OF THE CORONARY CIRCULATION POSSIBLE?}

Retrograde perfusion of the coronary circulation is possible because the veins of the heart have no valves, so cardioplegia given in a retrograde manner will reach the heart's capillaries and perfuse the myocardium. In fact, most perfusion beds in the body can be perfused in a retrograde manner. This is because, for all practical purposes, the only venous valves in the body are in the extremities. We frequently take advantage of this fact with retrograde cerebral perfusion [Cope 1996]. The feasibility of the perfusion of the spinal cord in a retrograde manner has also been established [Gangemi 2000]. All of these strategies allow for the delivery of protective solutions in a manner that obviates the need to have arterial access during operations. In addition, retrograde perfusion can facilitate the delivery of organ specific drugs in higher doses than could be used systemically.

WHEN SHOULD RETROGRADE CARDIOPLEGIA BE CONSIDERED?

- When there is aortic insufficiency (AI)

- During the repair of aortic dissections when AI may be present and when the coronary ostia may be involved in the dissection

- If complex, diffuse obstructive coronary artery disease is present

- During reoperations (to wash out particulate emboli from old grafts) [Gundry 1992]

- For heart transplants

- When there is (or might be) air in the coronaries
In addition to these particular situations, there is some value in the "same way every day" concept, meaning that if you get used to a strategy, it will be easier to use it when it's necessary.

\section{WHICH AREAS ARE BEST PROTECTED BY RETROGRADE CARDIOPLEGIA?}

The left side of the heart generally gets more of the cardioplegia administered via the retrograde route than does the right side. This is true because the retrograde catheters that are most commonly used are usually positioned as far into the coronary sinus (CS) as is feasible, primarily so that they won't become dislodged with manipulation of the heart or by the flow of the cardioplegia into the sinus, which can push a catheter back. The proper position of the catheter is between the middle cardiac vein and the great cardiac vein, as shown in Figure 1.

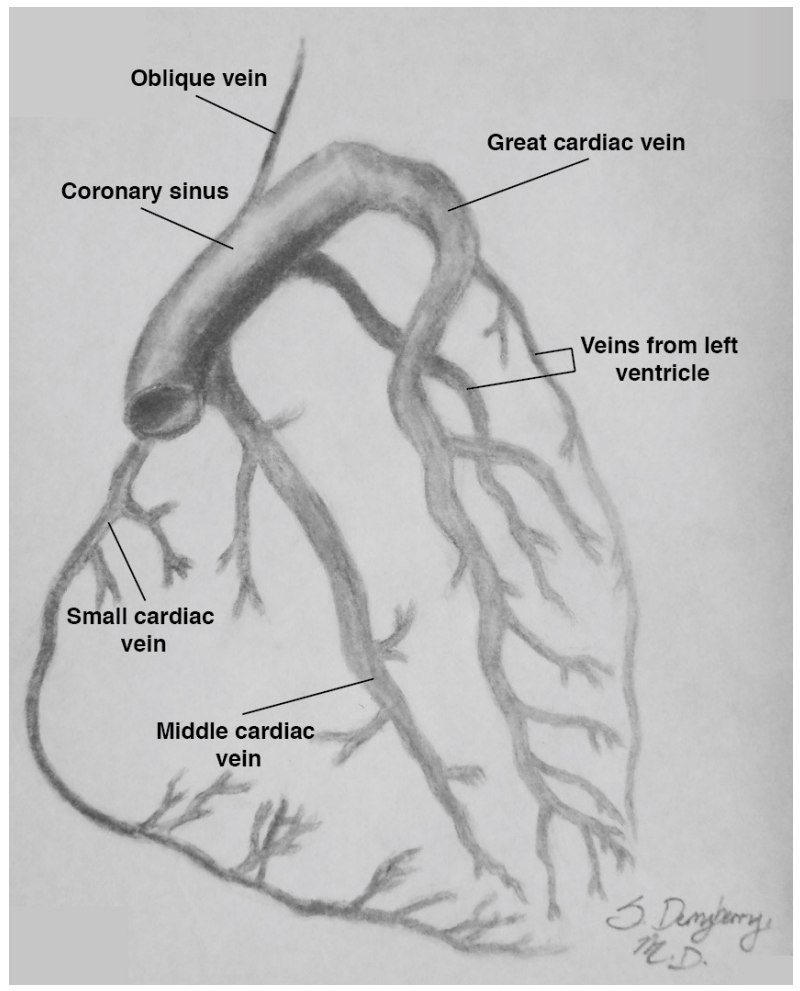

Figure 1: The venous anatomy of the heart, as viewed from the back of the heart, illustrating the usual position of a retrograde coronary sinus catheter between the middle cardiac vein and the great cardiac vein. 
The fact that the veins of the left side of the heart get more of the retrograde cardioplegia than do the veins of the right side often influences coronary grafting strategies. For instance, most surgeons graft the right coronary artery (RCA) first, if an RCA graft is needed. The fact that the right ventricle may get less protection than the left ventricle may also lead to the consideration of other cardiac protection strategies, including the use of bi-caval cannulation to lessen the contact of warm blood with the right atrium (RA) and right ventricle, and direct infusion of cardioplegia into the RA, which will be discussed below.

\section{CANNULATING THE CORONARY SINUS}

- The CS is almost always cannulated through the right atrium (though it is possible to cannulate the CS directly in some cases, as will be discussed later).

- A pursestring is placed in the RA, fairly anterior and superior on the RA wall, but inferior to and well away from the venous cannulation site. That is, the pursestring should not be placed low on the RA, as it is easier to maneuver the catheter in the RA and direct it towards the CS orifice when the cannulation site is further from the CS.

- This pursestring, like all pursestrings, should have more suture on the surface of the RA than inside. This is because it is the suture on the surface that compresses the atrial wall, whereas the suture on the inside merely holds the suture in place. The pursestring should also have at least four "bites" (and certainly not just two, which is a box stitch and may not adequately close the atriotomy when the catheter is removed). The suture is controlled with a Rummell tourniquet and tied to the catheter once it has been positioned. One must avoid tying this securing tie too tightly, as it can obstruct the pressure-monitoring lumen, which is a not uncommon problem.

The catheter shown in Figure 2 is stiff enough to be controllable, while being flexible enough that it is unlikely to injure the CS or the RA. Additionally, should it go through the tricuspid

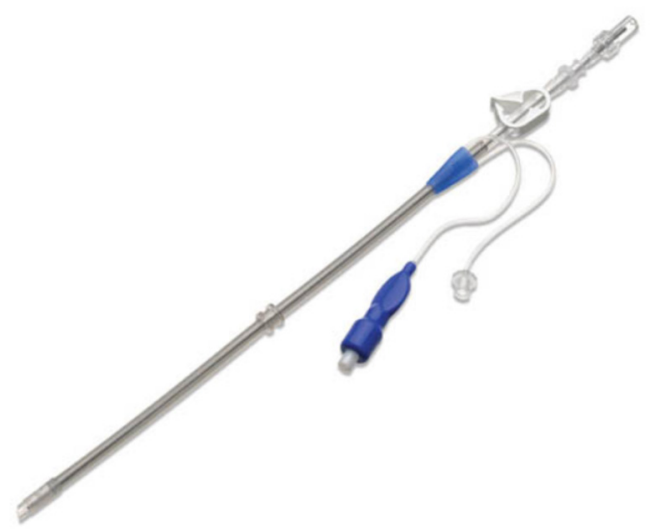

Figure 2. A picture of a commonly used CS catheter.

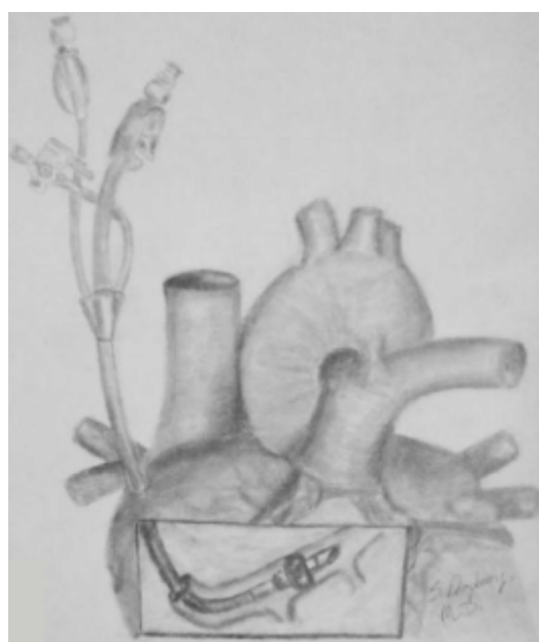

Figure 3: The catheter entering the coronary sinus.

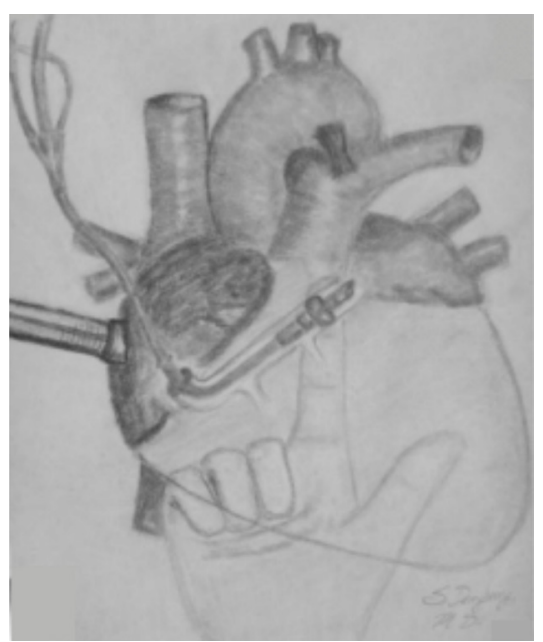

Figure 4: One hand should be placed behind the heart to feel the catheter entering the CS and getting it into proper position.

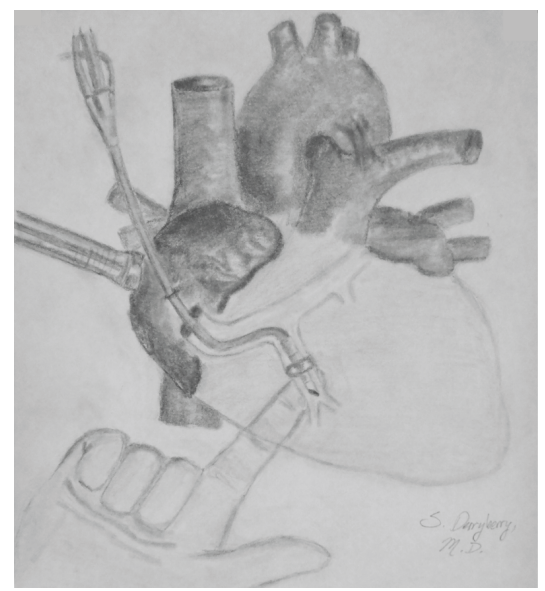

Figure 5: And, you want to avoid having the catheter end up in a tributary of the CS, as depicted here. 


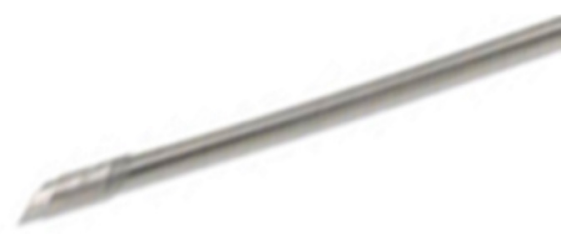

Figure 6: The beveled and relatively soft end of the catheter.

valve, it is unlikely to perforate the right ventricular free wall [Abramson 1998]. This most certainly can happen, especially in a patient with a fat-encased heart, which makes the catheter difficult to feel when it is being positioned. The catheter should be gently manipulated as far into the CS as feasible.

The end of the catheter is beveled (Figure 6). One should take advantage of this bevel by twisting or spinning the cannula as it is advanced into the CS, as doing so will lessen the chance that the tip will get "snagged" in a branch.

It is quite a bit easier to get the catheter into the CS from the left side of the table than from the right, so if it is a struggle to get the catheter into proper position, one should ask his or her first assistant to give it a try (or move to the left side oneself). The catheter should also be kept straight, not curved, as one will have a much better idea of where it is being directed when it is straight. It is also considerably harder to get the catheter to drop into the CS when it is curved than when it is straight. The catheter is flexible enough to conform to the anatomy of the CS.

Rarely, there will be a Thebesian valve that obstructs the CS ostium (Figure 7). Of course, if the RA is not open, it won't be possible to tell if such a valve is present. However, the CS can almost always be cannulated. One trick to remember is that one can insert one's left index finger into the RA through the pursestring in the RA appendage for the venous cannula. This finger can be used to guide the tip of the catheter into the CS (Figure 8) [Bahn 1991].

The catheter should always be positioned prior to going on cardiopulmonary bypass and prior to cross-clamping, because the CS is full under those conditions and is, therefore, larger. The heart should not be lifted to position the catheter, as it is

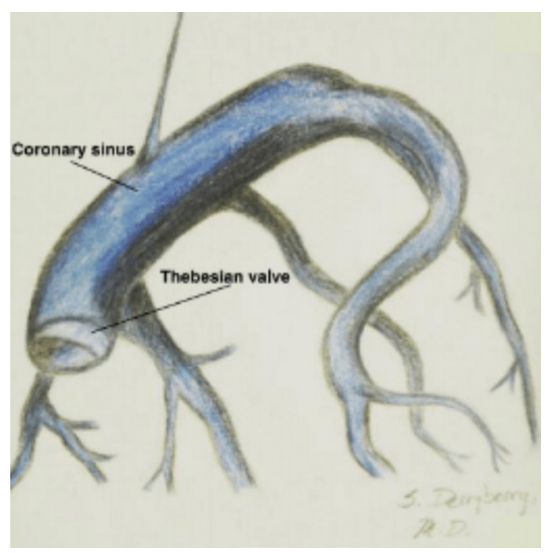

Figure 7: A Thebesian valve partially covering the CS ostium.

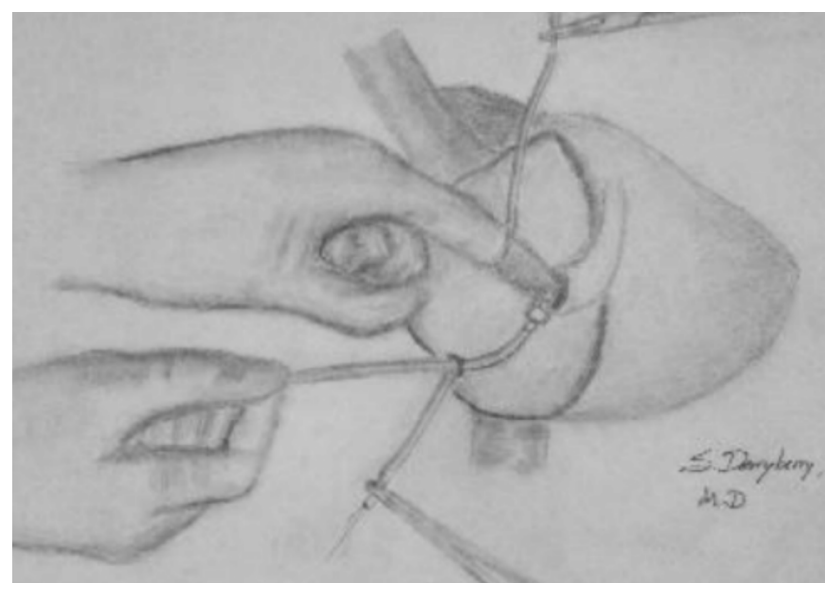

Figure 8: Directing the catheter into the CS with finger through atrial pursestring.

much easier to position the catheter with the heart in its normal position.

One should remember to feel for the catheter again when cross clamping and starting the delivery of cardioplegia. Placing one's hand behind the heart at this point will accomplish three things:

- One can be sure that the retrograde catheter is still in place

- One can feel for distention of the left ventricle

- One can stir around any cold water that might have been poured on the heart when starting the cardioplegia

Remember that one also needs to see the CS pressure rise at this point. (More on monitoring the pressure in the CS later.)

\section{AN ANATOMICAL VARIANT THAT PRECLUDES THE USE OF RETROGRADE CARDIOPLEGIA}

One cannot use retrograde cardioplegia when there is a persistent left superior vena cava (unless one is able to encircle and occlude this structure). In fact, a fairly frequent question on written exams that is related to this issue revolves around the issue of finding that the pressure in the sinus does not rise while infusing cardioplegia with a retrograde CS catheter in place.

\section{HOOKING UP THE RETROGRADE CATHERTER} TO THE CARDIOPLEGIA SYSTEM

An "octopus" multi-head rig should be used to hook up the antegrade and retrograde catheters, and to perfuse vein grafts as they are constructed (Figure 9).

It makes sense to hook up the arm with the blue clip to the retrograde catheter, as that means "blue goes to blue" (the retrograde catheter has blue components, as shown in Figure 2). It makes sense to routinely use the controlling clip closest to the heart (that is, the clip on the retrograde cannula), as the more proximal clip on the octopus itself may get covered by 


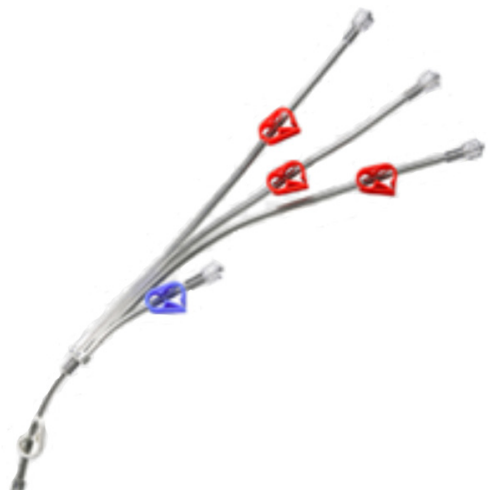

Figure 9: The multi-head 'octopus' device

towels while sewing coronary anastomoses or putting in valve stitches. It is necessary to de-air the retrograde catheter prior to hooking it up. The method used to de-air the cannula is to occlude the proximal CS with one's finger so that the CS blood flows backwards through the catheter and fills it. This blood is very dark, as the heart is the only organ that maximally extracts oxygen from the blood.

\section{ASSURING THAT THE CARIOPLEGIA IS FLOWING INTO THE CORONARY SINUS}

The monitoring port of the retrograde catheter needs to be hooked up to a high pressure line. Often, the anesthesiologists will hook up this line by exchanging it for the line used to monitor the Swan Ganz catheter, since the Swan won't be used while on bypass. At this point, it is a good idea to zero the line. The exact pressure is not very important. However, one must see the pressure rise with each infusion of the cardioplegia (and fall when the cardioplegia is turned off). If the pressure doesn't rise, something is awry, most likely indicating that the catheter has been displaced from the CS.

Once the aortic X-clamp has been applied and the retrograde cardioplegia has been started, one should see a rise in the pressure on the screen. If the pressure does not rise, the catheter may not be in proper position, or the clips may not be properly opened on the cardioplegia lines. Other possible reasons for a failure of the pressure to rise may be that the pressure monitoring line wasn't hooked up correctly or, occasionally, that the tie around the Rummell tourniquet and the retrograde catheter is tied too tightly. The CS can be occluded with one's finger, and if the CS pressure on the screen rises, one may need to put a bit more water into the balloon at the tip of the catheter.

Other methods to assure that proper retrograde delivery of the cardioplegia is occurring are to look for distention of the veins of the heart and to see that the veins have red blood in them. If the aorta has been opened (as in an AVR), one should also look for dark blood flowing from the coronary ostia (especially from the left coronary, because, as noted earlier, retrograde perfusion will preferentially go to the left side of the heart). Finally, it is comforting to see the heart arrest rapidly, showing proper cardioplegia delivery.

It is also important to know that one can, in fact, give antegrade and retrograde cardioplegia at the same time (a strategy sometimes called the "AC/DC" infusion). The heart will not "explode" when giving both antegrade and retrograde at the same time, as the Thebesian veins of the heart are robust enough to drain the cardioplegia adequately. The best sequence for starting the cardioplegia delivery is to start the antegrade cardioplegia first to ensure that it is flowing well and that there is no left ventricular distention. Then, the retrograde cardioplegia can be added, so that both antegrade and retrograde are running simultaneously.

\section{AVOIDING INJURY TO THE CORONARY SINUS}

It is important to realize that the CS can be injured by the CS catheter. Injury rarely happens with the Medtronic catheter, but some catheters have stiffer inner obturators or sharper tips, which can make injury more likely. The CS can also be injured by over-distention of the CS catheter balloon, so attention to this issue is warranted with catheters that do not have self-inflating balloons.

\section{REPAIRING AN INJURED CORONARY SINUS}

It has been said that an injured CS can be ligated or oversewn, but that is not always true, especially if the CS is narrowed or occluded near where it drains into the RA. It is better to repair an injured CS directly, if possible. To do this, one must dissect down on the CS so that the tear is visible and so that a patch of pericardium or vein can be sewn onto the hole. This approach should virtually always be done with the aortic cross clamp on, so that blood isn't pouring out of the injured spot and so that the heart is decompressed.

If, for some reason, visualizing the hole is not feasible, a patch of pericardium can be "glued" over the area with BioGlue. (In an earlier era, some surgeons kept tubes of Crazy Glue in their lockers for this purpose, but once BioGlue became available, it became more straightforward to use this commonly available adhesive for this purpose. Dermabond can also be used.) Some have advocated merely packing a lap pad over the area, with a plan to remove it the next day when the perioperative coagulopathy has subsided. An even more colorful strategy that has been described is to fill a glove with saline and to position it under the bleeding site, again with a plan to remove it the following day. The reason that these "minimal repairs" may work is that the pressure in the CS is low under normal conditions.

\section{USING THE RETROGRADE CORONARY SINUS CATHETER AT THE END OF THE OPERATION}

In most cardiac surgical operations, one can run warm blood through the CS catheter while performing the usual end-of-operation "chores", such as creating proximal vein graft anastomoses or closing the aortotomy in an aortic valve operation. Antegrade warm blood can also 
be run simultaneously during this time by opening the bulldog clamp on internal mammary artery grafts and running warm blood through the vein grafts that haven't yet been sewn to the aorta by keeping them hooked up to the multi-head octopus. This strategy provides plenty of oxygenated blood to an empty beating heart and will wash out residual cardioplegia and metabolites. (The warm, beating, empty heart needs only about $10 \%$ of the oxygen that a working heart requires.) The heart will almost always spontaneously defibrillate when perfused in this way, which "cannot be a bad sign." One will want to be sure that the heart is relatively empty so that once the heart starts to beat, blood will not be ejected through open incisions or proximal vein graft sites in the ascending aorta that have not yet had their veins sewn to them.

\section{THE TIMING OF REMOVAL OF THE RETROGRADE CORONARY SINUS CATHETER}

Even after stopping the flow of cardioplegia or warm blood through the catheter, the catheter should be left in place with the balloon deflated. After all, it is a lot easier to keep it in place than it is to replace it later if, for some reason, one should need to re-clamp and re-administer cardioplegia (for instance, if there is a paravalvular leak after an aortic valve replacement that one wants to look for, or if there is bleeding from a graft on the back of the heart).

\section{REMOVING THE RETROGRADE CATHETER}

Of course, the first step in removing the retrograde catheter will be to ensure that the balloon is deflated. Then, the atrial pursestring is tightened using the Rummell tourniquet. When the timing is right, the purse string can be tied down. A supplemental stitch can be placed to reinforce the pursestring. Such a reinforcing stitch might benefit from pledgets, especially if the atrial wall is thin. One can even consider using the purse string for an atrial wire site (with a completely stripped pacing wire that can be easily pulled out postoperatively).

\section{METHODS TO SUPPLY RETROGRADE PERFUSION TO MORE OF THE HEART}

A snare can be placed around the CS near the site of the entry of the CS into the RA (just medial to the inferior vena cava) (Figure 10). After this stitch is placed, one can pull the inflated balloon on the tip of the catheter back against the pursestring. This technique was first described by Calcaterra and Salerno at the University of Miami, and later by Gabbieri [Gabbieri 2009; Calcaterra 2007]. This snare is best created by using a 3-0 or a 2-0 Prolene on a large needle (Figure 11).

In some cases, a retrograde catheter can also be placed directly into the CS from the outside of the heart, if necessary. One will need to be able to see the CS well and to be able to put a pursestring into the area, through which the catheter can be inserted. The downside to this strategy is that the CS could be narrowed when the pursestring is tied down.

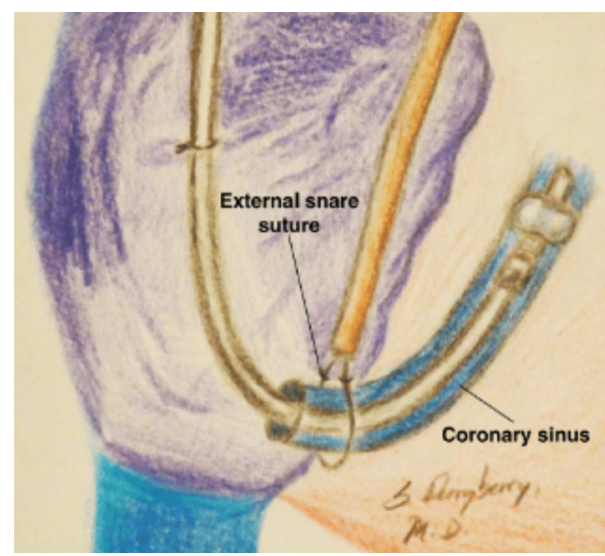

Figure 10: Snaring the proximal CS

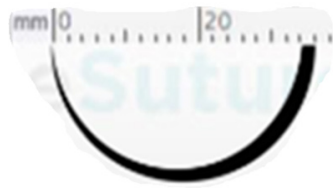

Figure 11: An MH needle suitable for encircling the proximal CS.

If the RA has been opened (such as when repairing the tricuspid valve, using a bi-atrial approach to the mitral valve, or in a heart transplant), a retrograde catheter can be placed directly into the coronary ostium. It is usually necessary to put a pursestring around the ostium, which will be removed later, of course (Figure 12).

\section{INFUSING CARDIOPLEGIA DIRECTLY INTO THE RIGHT ATRIUM}

Cardioplegia can also be infused directly into the RA, which will, of course, allow flow into the coronary sinus and all of its branches, as well as into the Thebesian

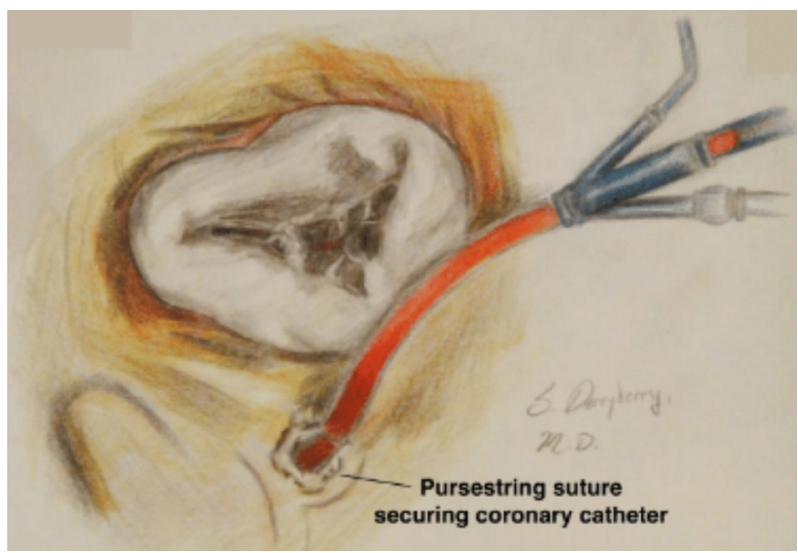

Figure 12: A CS catheter placed into the CS ostium in the right atrium. 


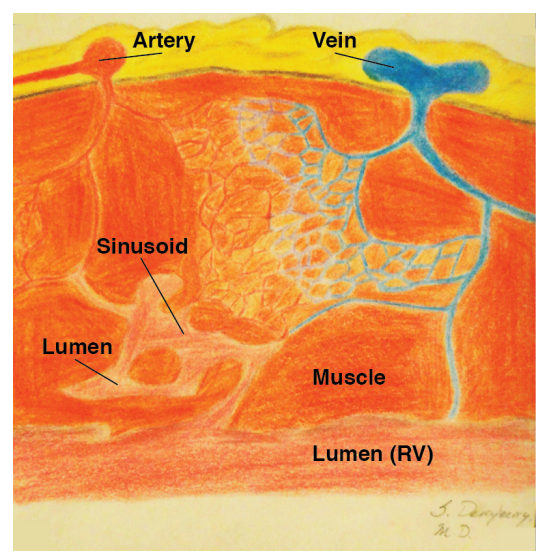

Figure 13: The Thebesian veins of the heart.

veins which drain directly into the RA (and, to some degree, into the ventricles, especially the right ventricle) (Figure 13).

This strategy was popularized by Alain Carpentier and his colleagues in the early days of mitral valve repair surgery [Fabiani 1986]. For this technique to work, one must, of course, have the cavae taped. It is also necessary to occlude the pulmonary artery (PA) while the cardioplegia is being infused. Occluding the PA can be easily accomplished by passing an umbilical tape through the transverse sinus and using it to snug the PA against the aorta intermittently. This technique is easier than clamping the PA (Figure 14). Dr. Carpentier used this strategy almost exclusively for his mitral valve repair work, as well as for other operations, such a coronary artery bypass grafting.
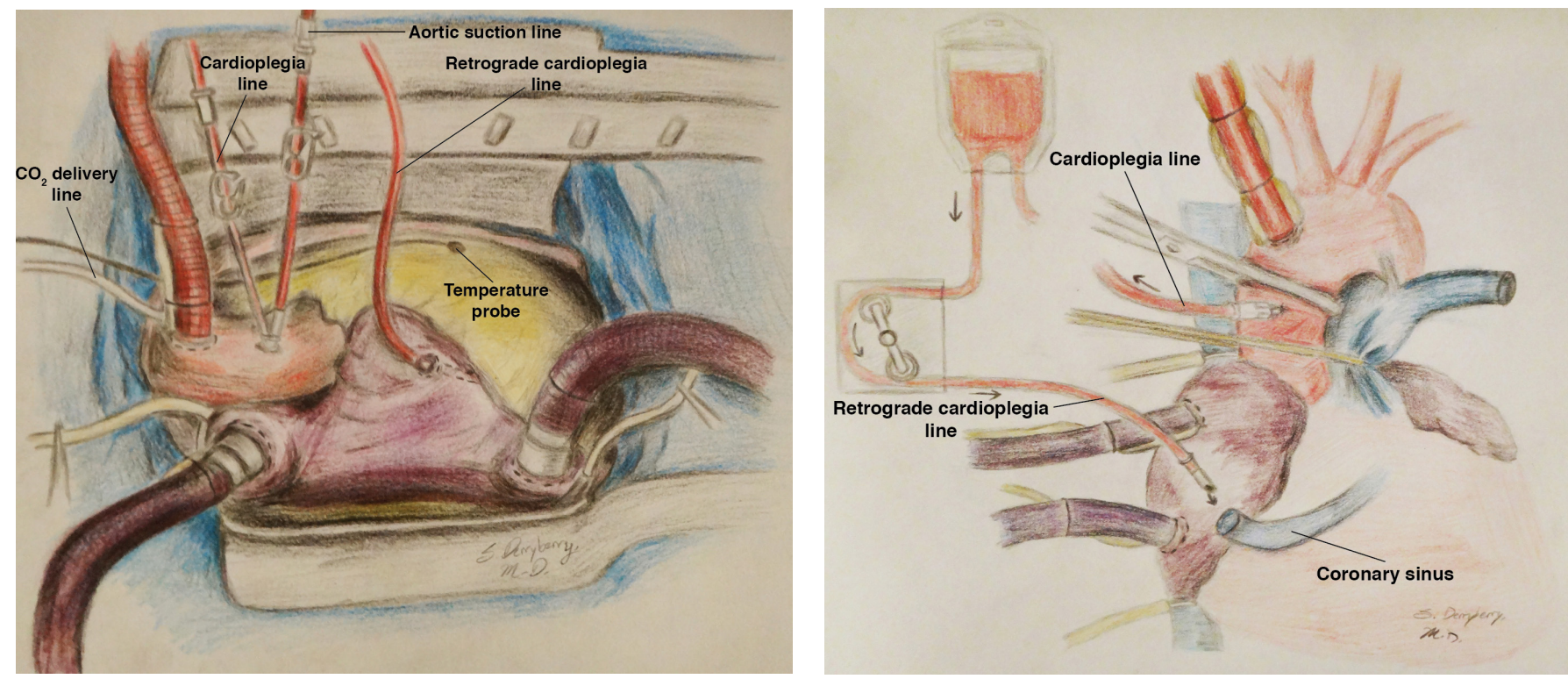

It is possible to position a retrograde CS catheter in minimally invasive cases (from the internal jugular vein in the HeartPort system or from the RA, but without a hand behind the heart, in other minimally invasive cases) by using wave forms on the monitoring line to direct the catheter into the CS. The wave form one will look for is sort of an arterial wave form, which is created by the full, perfused, beating heart (Figure 15). Obviously, this approach is challenging on a good day, and is sometimes simply not feasible.

\section{CONSIDERATIONS WHEN USING RETROGRADE CARDIOPLEGIA WHEN A LEFT VENTRICULAR PACING LEAD IS IN THE CORONARY SINUS}

Placing a retrograde cardioplegia cannula into the CS when a left ventricular pacing lead is in place can present a challenge, since the catheter may not fit into the CS and since the lead could be displaced. There are pediatric retrograde catheters that are available and can be used under these circumstances. One can also consider Dr. Carpentier's technique of infusing cardioplegia directly into the RA, as described above, when a left ventricular pacing lead is in the CS. If one does attempt to place a retrograde catheter into a CS with a pacing lead in it, it may make sense to place an epicardial lead on the left ventricle and lead it to a subcutaneous pocket to be used later if necessary. It is possible to pass such a lead into a device pocket if it is opened up by leading the lead out of the chest and into the device pocket through the second intercostal space.

\section{PLACEMENT OF RETROGRADE CORONARY SINUS CATHETERS IN MINIMALLY INVASIVE CASES}

Figure 14: Retrograde Cardioplegia through the Right Atrium. 


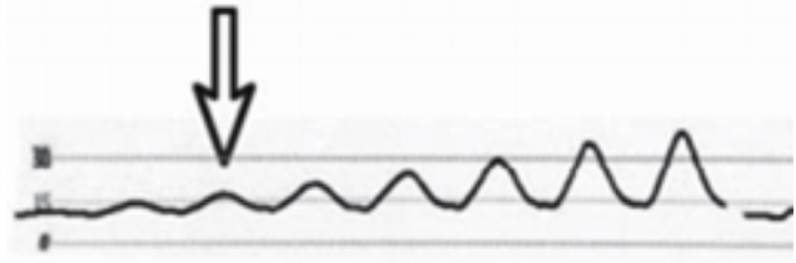

Figure 15: The change in waveform as the catheter enters the CS, going from an atrial wave form to a 'ventricularized' wave form.

\section{TROUBLESHOOTING THE CARDIOPLEGIA} SYSTEM WHEN SOMETHING SEEMS AWRY

The first consideration is whether to take the aortic cross clamp off to allow normal coronary perfusion to resume while the system is checked out in a stepwise manner.

Next, start at the pump and work back towards the heart.

I. Check the equipment and lines to check to see if:

a) the cardioplegia pump head isn't working.

b) the cardioplegia line is kinked somewhere.

c) a clamp is still on one of the cardioplegia lines.

II. Check to see if something is awry in the field, such as:

a) the aortic clamp is not across the aorta.

b) the retrograde catheter has been dislodged.

c) the retrograde balloon is not sufficiently inflated.

d) the monitoring line for the retrograde is not turned on and not being displayed on the monitor.

e) the tie around the Rummell tourniquet is too tight (obstructing the monitoring lumen on the retrograde cannula).

\section{THE CHECKLIST FOR BLEEDING PRIOR TO FINISHING UP}

One should always have a routine for checking for bleeding sites at the end of the operation, and this checklist should include checking the retrograde site, of course.

\section{SUMMARY}

Retrograde cardioplegia can be a very useful strategy for optimizing cardiac protection, and the techniques necessary to use this strategy optimally, which have been summarized here, have not always been clearly delineated in an organized manner.

\section{ACKNOWLEDGEMENTS}

The title of this essay is at least tangentially related to the eighth and last studio album by the British rock band, Led Zeppelin. The band said that the title was chosen as a sort of protest against a tax law that the British government had created in the 1970's which prompted the band to perform only outside Britain for a time, hence requiring them to make this album available through "the back door."

The original art work in this treatise was created by Dr. Stephen Derryberry, who is a Surgery Resident at Vanderbilt University. The other figures were derived from stock images freely available on-line.

\section{REFERENCES}

Abramson DC, Giannoti AG. 1988. Perforation of the right ventricle with a coronary sinus catheter during preparation for minimally invasive cardiac surgery. Anesthesiology 89:519-21.

Bahn CH. 1991. Simplified placement of retrograde cardioplegic catheter. Ann Thorac Surg 52:879-80.

Calcaterra D, Ricci M, Salerno T. 2007. A new technique for perfusion of the heart during aortic valvular surgery on a beating heart. J Card Surg 22:432-3.

Cope J, Tribble R, Komorowski B, et al. 1996. A simple technique for retrograde cerebral perfusion. J Card Surg 11:65-7.

Fabiani J, Deloche A, Swanson J, et al. 1986. Retrograde cardioplegia through the right atrium. Ann Thorac Surg 41:101-2.

Gabbieri, D, Pedulli, M, Bianchi, T, et al. 2009. External security stitch for retrograde cardioplegia cannula. J Card Surg 24:429-30.

Gangemi J, Kern J, Ross S, et al. 2000. Retrograde perfusion with a sodium channel antagonist provides ischemic spinal cord protection. Ann Thorac Surg 69:1744-9.

Gundry S, Razzouk A, Vigesaa R, et al. 1992. Optimal delivery of cardioplegic solution for "redo" operations. J Thorac Cardiovasc Surg 103:896-901.

\section{ADDITIONAL RESOURCES}

Berne, RM. 1964. Regulation of coronary blood flow. Physiological Reviews 44:1.

Burton, AC. 1965. Physiology and Biophysics of the Circulation. 2nd Edition. ISBN-13: 978-0815113645

Rudis E, Gates R, Laks H, et al. 1995. Coronary sinus ostial occlusion during retrograde delivery of cardioplegic solution significantly improves cardioplegia distribution and efficacy. J Thor Cardiovasc Surg 109: 941-7. 